\title{
Predictive inference on cytoplasmic and mitochondrial thioredoxin peroxidases in the highly radioresistant Lepidopteran insect Spodoptera frugiperda
}

\author{
Shashank Hambarde ${ }^{1}$, Ragothaman M. Yennamalli ${ }^{2,3}$, Naidu Subbarao ${ }^{2}$, Sudhir Chandna*1 \\ ${ }^{1}$ Natural Radiation Response Mechanisms Group, Division of Radiation Biosciences, Institute of Nuclear Medicine and Allied Sciences, \\ Brig. S.K. Mazumdar Road, Delhi -110054, India; ${ }^{2}$ Centre for Computational Biology and Bioinformatics, School of Information \\ Technology, Jawaharlal Nehru University, New Mehrauli Road, New Delhi 110067 India; ${ }^{3}$ Present address: 1032 Crop Genome Informatics \\ Laboratory, Department of Genetics, Development and Cell Biology, Iowa State University, Ames, Iowa 50011-3260 USA; \\ Sudhir Chandna E. mail - sudhirchandna@yahoo.com;*corresponding author
}

Received December 09, 2009; revised December 28, 2009; accepted February 08, 2010; published March 31, 2010

\begin{abstract}
:
Lepidopteran insects show remarkable resistance to radiation and chemical stress than insects of other orders. Despite this, the antioxidant machinery of insects of this order is poorly understood. Recently we demonstrated the significance of cytoplasmic NOS and a stronger mitochondrial antioxidant enzyme system in the stress-resistance of Lepidopteran insects. In the present study, we hypothesize two thioredoxin peroxidase orthologues (Sf-TPx1 and Sf-TPx2) in Lepidopteran insect Spodoptera frugiperda and demonstrate their structural/functional features important for cellular antioxidant activity and stress resistance. Results show a higher mitochondrial localization score (WoLFPSORT) of Sf-TPx2 (mitochondria-18.0, cytoplasm-7.0, nucleus-4.0) than its Drosophila orthologue Jafrac2 (secretory-30.0; mitochondria/nucleus/cytoplasm-no signal), which is important for antioxidant activity, and a higher cytoplasmic localization score of Sf-TPx1 (mitochondria-no signal; cytoplasm-22.0; nucleus-3.5) than the Drosophila Jafrac1 (mitochondria-17; nucleus11; cytoplasm-no signal). Structural modeling data show certain motifs present in Jafrac1 and Jafrac2 that affect active site conformation and separate cysteine residues at distances not suitable for disulphide bridge formation (5.21 ; $_{5}$.73 $\AA$ ). These motifs are absent in Sf-TPx 1 and Sf-TPx2, yielding shorter distance $(2.01 \AA ; 2.05 \AA)$ between the cysteine residues suitable for disulphide bridge formation. Taken together, the disulphide bridge as well as mitochondrial and cytoplasmic localization are crucial for peroxidatic activity of TPx's. Therefore, we hypothesize that the Spodoptera TPx's offer potentially stronger anti-oxidant activity than that of Drosophila orthologues, and may contribute in the high radioresistance of Lepidopteran insects.
\end{abstract}

\section{Background:}

Exposure to stress agents such as ionizing radiation that cause formation of reactive oxygen and nitrogen species leads to activation of cellular anti-oxidant systems for protecting cells from damage or apoptosis. One of these anti-oxidant systems is the thioredoxin system containing thioredoxin (Trx, a small protein with two redox active cysteine residues in its active center) and two enzymes thioredoxin peroxidase (TPx) and thioredoxin reductase (TrxR). In response to oxidative stress, oxidation of Trx by the TPx enzyme is used for reduction and detoxification of hydrogen peroxide, peroxynitrites and a wide range of organic hydroperoxides $(\mathrm{ROOH}+2 \mathrm{e}-\rightarrow \mathrm{ROH}+\mathrm{H} 2 \mathrm{O})$. As a result, TPx may play important role in countering the cellular oxidative stress. For example, overexpression of certain isoforms of TPx as observed in several cancers [1-5] has been shown to inhibit stress-induced increase in intracellular hydrogen peroxide [6] and is also associated with tumour resistance to radiotherapy [7] or chemotherapy [8]

The TPx family of proteins is classified into three distinct classes (based on the nature of their active site), viz., 1-Cys TPx, atypical 2Cys TPx and typical 2-Cys TPx. While the 1-Cys TPx contains only one cysteine residue for peroxidation at the active site, the 2-Cys TPx's contain two cysteine residues. In Atypical 2-Cys TPx, the two cysteine residues, peroxidatic and resolving, are present on the same polypeptide. However in typical 2-Cys TPx dimerization is essential for formation of active site, since one of the monomers contains peroxidatic cysteine $\left(\mathrm{C}_{\mathrm{P}}\right)$ while the other contains resolving cysteine $\left(\mathrm{C}_{\mathrm{R}}\right)$ residue. The conformation of typical 2-Cys TPx is regulated by its oxidation status and intracellular oxidant level, and shifts between dimer and decamer conformation, the latter conformation being less efficient in peroxidase activity [9]. In the event of $\mathrm{H}_{2} \mathrm{O}_{2}$ flux, sulfenic acid on the peroxidatic cysteine residue $\left(\mathrm{C}_{\mathrm{P}}\right)$ at active site is oxidized to sulfinic acid ( $\mathrm{SOH})$. The sulfinic acid on $\mathrm{CP}$ then reacts with the conserved resolving cysteine $\left(C_{R}\right)$ to yield an intersulfide bond that is eventually reduced by thioredoxin reductase (TrxR). Since insects do not possess significant glutathione peroxidase activity, it is understood that TPx might play important role in the enzymatic removal of reactive oxygen species (ROS) [10]. While the role of TPx in elimination of $\mathrm{H}_{2} \mathrm{O}_{2}$ and other organic peroxides coupled with prevention of cell death has been demonstrated in insects, yet the nature and function of insect TPx is not generally very well understood.

We have been studying Lepidopteran insect cells since these cells display 50-100 times higher radioresistance than the mammalian/ human cells despite showing significant structural/ functional homologies. These are also evolutionarily closest to the human system amongst all the highly radioresistant organisms known. Apparently, reduced DNA damage [11] possibly contributed by soluble radioprotectors and chromatin organisation, enhanced DNA repair [12], resistance against radiation-induced apoptosis [11], and a stronger cytosolic [13] and mitochondrial [14] antioxidant machinery seem to play dominant role in the radioresistance of these cells. Reports available so far do not present a conclusive picture of the antioxidant machinery of Lepidopteran insect cells. While catalase and glutathione peroxidase activity was previously reported to be absent in these cells [15], activity/expression of superoxide dismutase, ascorbate peroxidase, glutathione reductase, glutathione peroxidase and catalase were reported later in Spodoptera littoralis [16]. Therefore, the antioxidant machinery of this highly radioresistant model system needs to be understood further. In our recent study on mitochondrial antioxidant system, the thioredoxin system of Lepidopteran insect Bombyx mori predicted a considerably higher mitochondrial localization potential (Mitoprot scores) compared to the human system [14]. In the present study, we describe the thioredoxin system in Spodoptera (Insecta; Lepidoptera); while utilizing the components of Drosophila thioredoxin system, i.e., thioredoxin ( $\operatorname{Trx})$, thioredoxin reductase (TrxR) and thioredoxin peroxidase (known as Jafrac1 \& Jafrac2) and identifying their orthologs available in S. frugiperda EST database. Possible implications in stress/radiation resistance are discussed.

\section{Methodology:}

\section{Sequence data retrieval and BLASTp}

Protein sequences of Trx, TrxR and TPx of various insect species were retrieved from GenBank by using appropriate key words. Drosophila Trx, TrxR and Jafrac1 \& 2 sequences retrieved from GenBank were used as query sequences in BLASTp of http://butterflybase.ice.mpg.de [17] against 'Spodoptera frugiperda 


\section{Bioinformation}

predicted proteins database'. The sequences showing lowest E values were selected for further analysis.

\section{Sequence analysis and secondary structure alignment}

Two sequences were selected as mentioned above and were aligned with Drosophila Jafrac1 and Jafrac2 using BioEdit (http://www.mbio.ncsu.edu/BioEdit) and Secondary structures mentioned in pdb files were manually depicted along with sequence alignment. TPx sequences from other insect species namely Bombyx mori, Apis mallifera ligustica and Helicoverpa armigera along with two putative Sf-TPx and Jafrac1 and 2 were aligned by using ClustalW in BioEdit to study sequence conservation across species of insects.

Intracellular localization signal prediction

Sequence of TPx from various insect species derived from GenBank as well as EST database BLAST results was used for prediction of intracellular localization signal score by the online tool WoLF PSORT [18] available on http://wolfpsort.org.

Modeling monomer and dimer

Protein sequences of Jafrac1 \& 2 and putative Sf-TPx 1 \& 2 were used to generate tertiary structure by Modweb. Dimers were generated by superimposing modeled monomer structures onto individual monomers of known dimer structures from PDB using Combinatorial extension program (http://cl.sdsc.edu/ce.html). 1QMV for fully folded (FF) conformation and 1QQ2 for locally unfolded conformation [19] was used. We used UCSF Chimera [20] for building the multimer and Insight II (www.accelrys.com) for optimizing the structure and removing the clashes. To remove the steric clashes between the side chains of the mutated and neighboring residues, energy minimization was performed for 10000 steps using conjugate-gradient method employed in the Discover3 module of Insight II. The force field used to set the potentials was CVFF force field. Swiss PDB Viewer was used to visualize the active site of dimer model and measurement of distance between cysteine residues of active site was calculated by using PyMol.

\section{Protein-protein interaction study}

To study the interaction of Jafrac1 \& 2 with DIAP1 and Sf-TPx1 \& 2 with Sf-IAP-1, we used BIR2 domains of both DIAP1 and SfIAP-1 respectively. Tertiary structures of BIR2 domains predicted by SwissModel were used for protein-protein interaction on PatchDock [21]. The docking results were analyzed and structures with highest scores were used for calculation of $\mathrm{E}_{\text {Total }}$ by Hex $5 \mathrm{a}$.

\section{Discussion}

Two isoforms of typical 2-Cys TPx detected in S. frugiperda As mentioned, we used the sequences of Drosophila Trx, TrxR and TPx (Jafrac1/ Jafrac2) as queries for search in 'Spodoptera frugiperda predicted proteins database' of Butterflybase (http://butterflybase.ice.mpg.de). BLASTp failed to show homology with Trx and TrxR (results not shown). However, orthologs of Jafrac 1 and 2 were identified with very high bit score. Jafrac1 best hit sequence (accession number SFP00903 3) showed an E-value of $1 \times 10^{-90}$ and identity of $82 \%$ (Sf-TPx 1$)$ and Jafrac 2 best hit (accession number SFP07707_2) showed an E-value of $6 \times 10^{-77}$ with an identity of $64 \%$ (Sf-TPx 2$)$. These results indicate the presence of two putative TPx's in S. frugiperda, viz., Sf-TPx1 and Sf-TPx2. Absence of any hits for Trx and TrxR only suggest that the non-exhaustive EST database still remains to identify such proteins in Spodoptera. Our ClustalW analysis of TPx sequences of some other insect species along with Jafrac1/2 and newly identified Sf-TPx $1 / 2$ showed that GGLG and YF motifs, active site cysteine residues $\left(C_{P}\right.$ and $\left.C_{R}\right)$ are well conserved in all insects investigated and that newly identified Sf-TPx1 \& 2 belong to the typical 2-Cys TPx family of proteins (Figure 1).

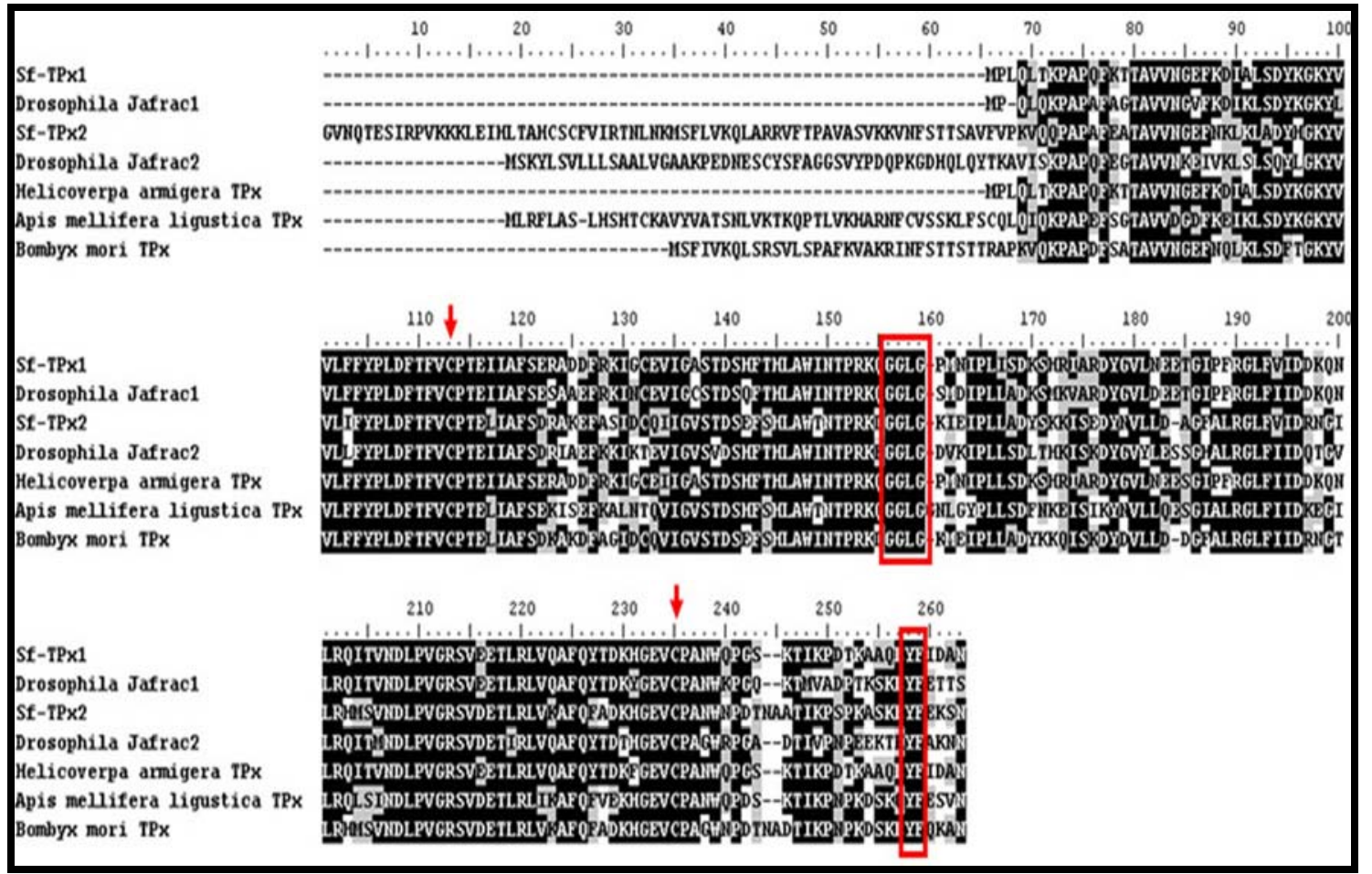

Figure 1: Multiple sequence alignment, using ClustalW, of amino acid sequence of thioredoxin peroxidases of various insect species indicate high conservation in C-terminal region (with black background). Box showing conserved GGLG and YF motifs and arrows showing conserved Cystein residues $\left(\mathrm{C}_{\mathrm{P}}\right.$ and $\left.\mathrm{C}_{\mathrm{R}}\right)$ of active site in various insect TPxs. Helicoverpa armigera (Cotton ballworm) Acc No. ABW96360.1, Bombyx mori Acc. No. NP 001040464.1, Apismellifera ligustica AAP93584.1, Drosophila Jafrac1 Acc. No. AAF42985.1, Jafrac2 Acc No. AAF42986.1 


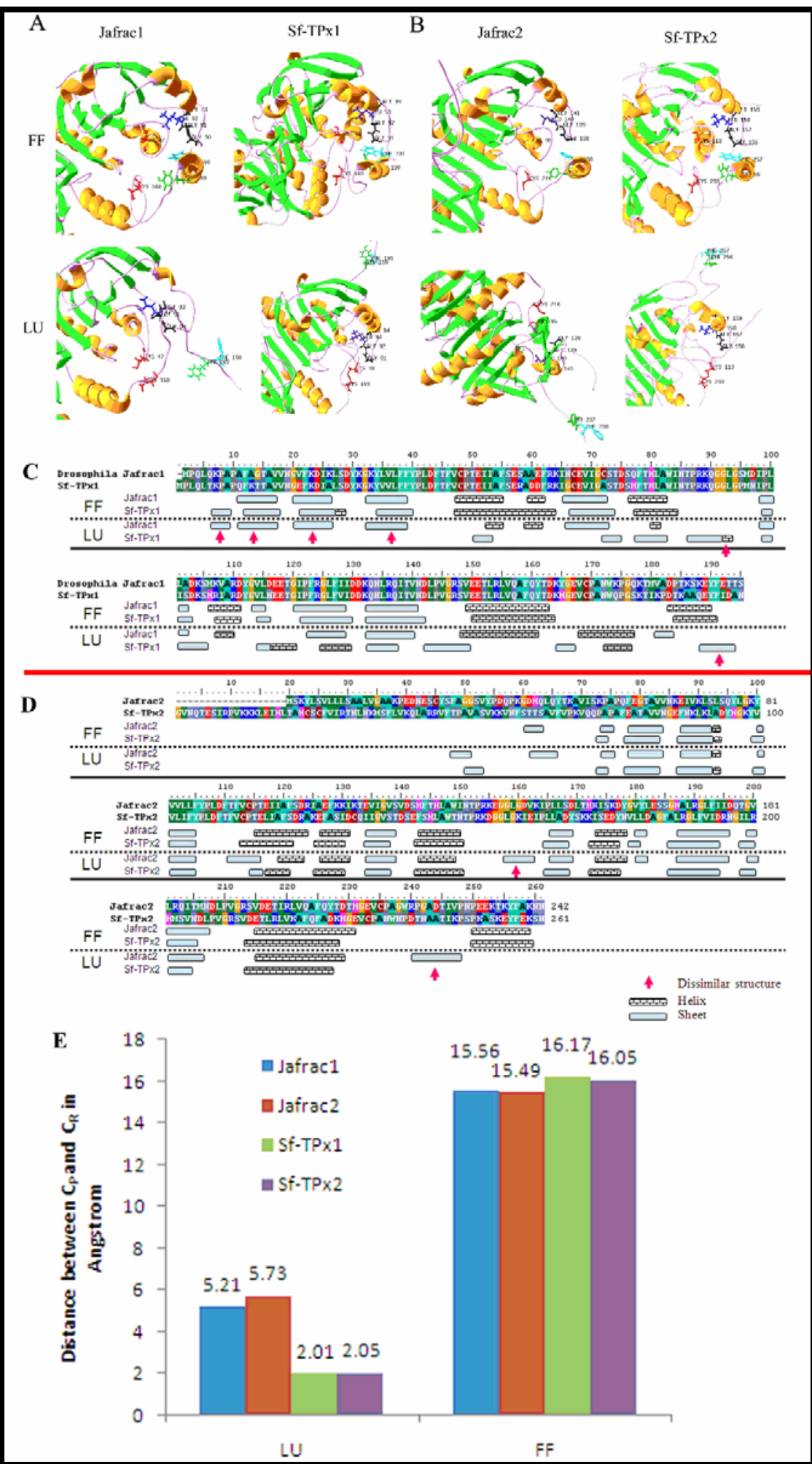

Figure 2: Comparison of active site conformation in modeled dimer structure of (A) Jafrac1 with Sf-TPx1 and (B) Jafrac2 with Sf-TPx2 in FF and LU conformation. GGLG and YF motifs (G - Black color, L - Blue, Y - Green, F - Sky blue) bury active site cysteine residues (in Red sticks) in FF conformation but not in LU conformation. Sf-TPx1 predicted to have disulphide bond between Cys49 of one subunit with Cys169 of other subunit of the dimer. (C, D) Primary and secondary structure alignment depicting comparison of helix and sheet structures in FF and LU conformation (derived manually from pdb files) of Jafrac1 versus Sf-TPx1 and Jafrac2 versus Sf-TPx2. The blue horizontal bars representing sheets and checkered horizontal bars representing helices. Areas with dissimilar structures are indicated with pink arrows. (E) Distance (in Angstroms) between cysteine residues $\left(\mathrm{C}_{\mathrm{P}}\right.$ and $\mathrm{C}_{\mathrm{R}}$ ) of active site in FF and LU conformation. 
Dimer models of Sf-TPx1 and Sf-TPx2 predict disulphide bond formation in $\mathrm{LU}$ conformation

We report herein the modeled dimer structures of newly identified Sf-TPx $1 \& 2$ as well as of Drosophila Jafrac1 \& 2 (Figure 2). Sequence identity for 1QMV monomers with Sf-TPx1 and Sf-TPx2 is $71.1 \%$ and $63.7 \%$, respectively; and $70.0 \%$ and $68.4 \%$ with Jafrac1 and Jafrac2. Sequence identity for 1QQ2 monomers with SfTPx 1 and Sf-TPx2 are $75.3 \%$ and $64.7 \%$; and $71.2 \%$ and $67.6 \%$ with Jafrac1 and Jafrac2, respectively. The length of alignment between template individual monomers and models after superimposition to generate dimers was found to be high as well (data not shown). The rmsd for 1QMV monomers and models of SfTPx1 and Sf-TPx2 was $1.4 \AA$ and $0.5 \AA$, while for the models of Jafrac1 and Jafrac2 it was $1.2 \AA$ and $0.5 \AA$, respectively. The rmsd for 1QQ2 monomers with models of Sf-TPx1 and Sf-TPx2 was 1.1 $\AA$ and $0.4 \AA$, and $1.0 \AA$ and $0.3 \AA$ with the models of Jafrac1 and Jafrac2, respectively. In agreement with the TPx's of other eukaryotes [19], these modeled dimer structures indicate that in the Fully Folded (FF) conformation, cysteine residues of active site $C_{P}$ and $\mathrm{C}_{\mathrm{R}}$ are buried under GGLG and YF motifs (Figure 2A, 2B) while being separated far away from each other (Figure 2E) to facilitate prevention of disulphide bridge formation and enzyme activity. In contrast, when these proteins are in Locally Unfolded (LU) conformation, the two cysteine residues come significantly close to each other (Figure 2E) and move towards the surface of protein (Figure 2A, 2B) in order to become available for the peroxidatic activity and to make the enzyme active. Comparison of distance between the cysteine residues (Figure 2E) revealed that $\mathrm{C}_{\mathrm{P}}$ (Cys48 in Sf-TPx1 and Cys113 in Sf-TPx2) of one monomer and $\mathrm{C}_{\mathrm{R}}$ (Cys169 in Sf-TPx1 and Cys233 of Sf-TPx2) of the other monomer are at a distance that is suitable for intersulphide bond formation $(2.01 \AA$ in Sf-TPx $1 ; 2.05 \AA$ in Sf-TPx2) in LU conformation. Therefore, both Sf-Tpx1 and Sf-TPx2 predict formation of intersulphide bond. Contrary to this, in Jafrac1 and Jafrac2 LU conformation, the active site cysteine residues are at a distance $(5.21$ $\AA$ in Jafrac1; $5.73 \AA$ in Jafrac2) (Figure 2E) that is higher than the natural distance required for disulphide bond $(2.03 \AA)$, and hence less likely to form a disulphide bridge. Therefore, it is very likely that Sf-TPx1 and Sf-TPx2 may have different peroxidatic activity than Jafrac1 and Jafrac2.

Secondary structure analysis of LU conformation supports disulphide bond formation in Sf-TPx's predicted from tertiary structure

To gain further insight of dissimilarities in the tertiary modeled structures, we aligned peptide sequence along with secondary structures predicted for the modeled dimer (LU and FF conformation) of putative Sf-TPx1 and Sf-TPx2 with those of Jafrac1 and Jafrac2, respectively (Figure 2C, 2D). In LU conformation, many $\mathrm{N}$-terminal residues of Jafrac1 are in sheet structure though same was not evident in Sf-TPx1. One of the cysteine residues $\left(C_{R}\right)$ within the active site of Jafrac1 is present in helix structure but not in Sf-TPx1. Two motifs (GGLG and YF) that are responsible for covering active site make a sheet-helix structure in LU conformation in Sf-TPx1 but not in Jafrac1 though both attain same structure in the FF conformation. Therefore, it appears that in Jafrac1, the formation of four extra sheets in N-terminus and the presence of $C_{R}$ in helix prevents S-S bond formation at the active site in LU conformation, which is not the case with Sf-TPx1. In Jafrac2, modeled LU conformation revealed that GGLG motif is in sheet structure and that there is an extra sheet at C-terminus which is dissimilar to Sf-TPx2; however in FF conformation, the Cterminal extra sheet disappears and GGLG motif attains same structure in both Jafrac2 and Sf-TPx2. These structural motifs may affect active site conformation of Jafrac 2 and result in increased distance between $C_{P}$ and $C_{R}$, preventing disulphide bridge formation. However, the absence of these structures in Sf-TPx2 favours disulphide bridge formation in its active site. Taken together, the analysis of secondary and tertiary structures indicates that the absence of certain structural motifs of Jafrac1 and Jafrac2 in both Sf-TPx 1 and Sf-TPx 2 may result in closely apposed active site Cys residues in these Sf-TPx's.
Spodoptera exhibits stronger antioxidant defence system than Drosophila

In Drosophila, Jafrac1 and Jafrac2 show distinct functions in antioxidant defence mechanism and apoptosis regulation. Jafrac1 localizes to cytoplasm and plays crucial role in the detoxification of ROS whereas Jafrac2 is present in endoplasmic reticulum and is rapidly released into cytoplasm on induction of apoptosis [22] When translocated to cytoplasm, Jafrac2 interacts with BIR2 domain of DIAP1 (Drosophila Inhibitor of Apoptosis Protein-1) and causes liberation of DRONC (Caspase). In this way Jafrac2 bridges between antioxidant system and apoptosis regulation. Since Jafrac1 and 2 are localized in different compartments of Drosophila cells we analyzed predicted localization signals of Sf-TPx1 and Sf-TPx2 by WoLFPSORT (Table 1 in supplementary material). Our analysis shows that Jafrac1 and Sf-TPx1 are localized to cytoplasm while Jafrac 2 exhibits secretary signals and Sf-TPx 2 resides mainly in the mitochondria. We have recently reported higher mitoprot score of Trx as well as TPx in the Lepidopteran insects as compared to insects of other orders [13]. In the present study, detailed analysis of localization of TPx revealed that in Lepidopteran insects, i.e., $B$. mori, H. armigera and $S$. frugiperda, TPx exists either in cytosol or in mitochondria which may result in stronger antioxidant defence system and resistance against stress induced death. However, in the Dipteran insect Drosophila, mitochondria are devoid of TPx, which in turn may enhance cellular sensitivity to oxidative stress.

Since BIR2 domain of DIAP-1 is known to interact with IAP binding motif (IBM) of Jafrac2 and contributes in regulation of apoptosis, we predicted the protein-protein interaction of DIAP1 BIR2 domain with Jafrac1 \& 2 and Sf-IAP-1 BIR2 domain with SfTPx $1 \& 2$. We used both LU as well as FF conformations of Jafrac1 $\& 2$ and Sf-TPx1 \& 2 for the interaction study. Our observations suggest that DIAP1:Jafrac2 (LU) complex (E total -388.46) and SfIAP-1: Sf-TPx1 (LU) complex (E total -341.91) have minimum $\mathrm{E}_{\text {Total }}$ (Table 2 in supplementary material). Since lesser $\mathrm{E}_{\text {Total }}$ is indicator of the most likely forming complexes, these results indicate that there are potential cross-talks possible between the antioxidant systems and apoptosis signaling in Spodoptera as well, which predict distinct mechanisms compared to Drosophila. While the Jafrac2 present in ER of Drosophila translocates to cytosol and interacts with DIAP-1 BIR2 domain upon apoptosis induction, regulation of interaction between Sf-IAP-1 BIR2 domain and the constitutively cytosolic Sf-TPx1 predicted here needs further investigation.

\section{Conclusion:}

In the present study, we report evidence of existence of two isoforms of TPx (Sf-TPx1 and Sf-TPx2) in Spodoptera frugiperda, and also demonstrate structural features that may be important in determining their antioxidant activity and apoptosis regulation. Each of these isoforms predicted different intracellular localization, viz., mitochondrial localization for Sf-TPx2 that is important for antioxidant activity, and cytoplasmic localization of Sf-TPx1 along with predicted interaction with Sf-IAP-1 important for regulating apoptotic activity. Models of both Sf-TPx1 and Sf-TPx2 show significantly higher possibility of formation of disulphide bridge at active site than their orthologues in Drosophila Jafrac1 and Jafrac2. Based on these observations, we hypothesize that the Spodoptera thioredoxin peroxidase might be a stronger anti-oxidant system as compared to that of Drosophila, and may potentially contribute in the relatively higher radioresistance of Lepidopteran insects. Since our protein-protein interaction study predicts that Sf-TPx1 localized in cytoplasm can actively interact with the anti-apoptotic Sf-IAP-1, we are further investigating its role in the regulation of Lepidopteran apoptosis.

References:

[1] D. Y. Noh et al., Anticancer Res (2001) 21: 2085 [PMID: 19566940]

[2] T. Yanagawa et al,. Cancer Lett (1999) 145: 127 [PMID: 19298244]

[3] T. Yanagawa et al., Cancer Lett (2000) 156: 27 [PMID: 10840156]

[4] V. L. Kinnula et al., J Pathol (2002) 196: 316 [PMID: 


\section{Bioinformation}

$11857495]$

[5] J. W. Chang et al., Biochem Biophys Res Commun (2001) 289: 507 [PMID: 11716502]

[6] S. W. Kang et al., J Biol Chem (1998) 273: 6297 [PMID: 9497357]

[7] S. H. Park et al., Clin Cancer Res (2000) 6: 4915 [PMID: 11156252]

[8] Y. M. Chung et al., Anticancer Res (2001) 21: 1129 [PMID: 11396151]

[9] T. Matsumura et al., J Biol Chem (2008) 283: 284 [PMID: 17974571]

[10] S. N. Radyuk et al., Biochem J (2003) 371: 743 [PMID: 12556226]

[11] S. Chandna et al., Int J Radiat Biol (2004) 80: 301 [PMID:15204707]

[12] T. M. Koval et al., Mutat Res (1978) 49:431 [PMID: 634308]

[13] C. Cheng et al., Mutagenesis (2009) 24: 259 [PMID:
19264841]

[14] S. Suman et al., Bioinformation (2009) 4:19

[15] Y. Wang et al., Free Radic Biol Med (2001) 30: 1254 [PMID: 11368923]

[16] M. E. Maffei et al., Plant Physiol (2006) 140: 1022 [PMID 16443697]

[17] A. Papanicolaou et al., Nucleic Acids Res (2008) 36: D582 [PMID: 17933781]

[18] P. Horton et al., Nucleic Acids Res (2007) 35: W585 [PMID 17517783]

[19] Z. A. Wood et al., Science (2003) 300: 650. [PMID: 12714747]

[20] E. F. Pettersen et al., J Comput Chem (2004) 25:1605 [PMID: 15264254]

[21] D. Schneidman-Duhovny et al., Nucleic Acids Res (2005) 33: W363 [PMID: 15980490]

[22] T. Tenev et al., EMBO J (2002) 21: 5118 [PMID: 12356728]

Edited by P. Kangueane

Citation: Hambarde et al., Bioinformation 4(9): 399-404 (2010) License statement: This is an open-access article, which permits unrestricted use, distribution, and reproduction in any medium, for noncommercial purposes, provided the original author and source are credited. 


\section{Bioinformation}

\section{Supplementary material:}

Table 1: Analysis of intracellular localization signal of Jafrac1, Jafrac2, Sf-TPx1 and Sf-TPx2 indicate that Jafrac1 and Sf-TPx1 are localized in cytoplasm, Jafrac2 in Drosophila has excretory signal while Sf-TPx2 localizes in mitochondria. Endoplasmic Reticulum (ER)

\begin{tabular}{llllll}
\hline & Cytoplasm & Nucleus & Mitochondria & ER & Excretory \\
\hline Jafrac1 & 17.0 & 11.0 & No Signal & No Signal & No Signal \\
Sf-TPX1 & 22.0 & 3.5 & No Signal & No Signal & No Signal \\
Jafrac2 & No Signal & No Signal & No Signal & No Signal & 30.0 \\
Sf-TPX2 & 7.0 & 4.0 & 18.0 & No Signal & No Signal \\
Bombyx TPx & No Signal & No Signal & 29.5 & No Signal & No Signal \\
Apis TPx & No Signal & No Signal & 29.5 & No Signal & No Signal \\
Helicoverpa TPx & 22.5 & 4.0 & No Signal & No Signal & No Signal \\
\hline
\end{tabular}

Table 2: Total energy $\left(\mathrm{E}_{\mathrm{T} o t a l}\right)$ values of the protein complexes of IAP's and TPx's indicating that Jafrac2 and TPx1 may interact with respective IAP's and can play important roles in apoptosis signaling.

\begin{tabular}{ll}
\hline Protein-protein interaction & $\mathbf{E}_{\text {Total }}$ \\
\hline DIAP1-Jafrac1 FF & -136.61 \\
DIAP1-Jafrac1 LU & -297.8 \\
DIAP1-Jafrac2 FF & -156.19 \\
DIAP1-Jafrac2 LU & -388.46 \\
Sf-IAP1-SfTPx1 FF & -164.96 \\
Sf-IAP1-SfTPx1 LU & -341.91 \\
Sf-IAP1-SfTPx2 FF & -203.76 \\
Sf-IAP1-SfTPx2 LU & -180.59 \\
\hline
\end{tabular}

